\title{
Electrochemical Characterization of the Powder Silicon Anodes Reinforced with Graphite Using Planetary Ball Milling
}

\author{
T. Cetinkaya, O. Cenher, U. Tocoglu, M.O. Guler and H. Akbulut \\ Sakarya University, Engineering Faculty, Department of Metallurgical \& Materials Engineering \\ Esentepe Campus, 54187, Sakarya, Turkey
}

\begin{abstract}
Mixed silicon/graphite anode materials were produced via mechanical milling process. The morphologies of mixed powders and electrodes were characterized via scanning electron microscopy and X-ray diffraction pattern. Electrochemical tests were performed by coin-type (CR2016) test cells. The cells were cyclically tested on a battery tester, and discharge capacities of produced anode materials were investigated with using constant current $300 \mathrm{~mA} / \mathrm{g}$ over a voltage range of $80 \mathrm{mV}-1.2 \mathrm{~V}$.
\end{abstract}

DOI: $10.12693 /$ APhysPolA.123.393

PACS: 61.72.uf, 81.05.uf, 81.20.Ev, 82.45.Fk, 82.47.Aa

\section{Introduction}

Lithium-ion (Li-ion) batteries are currently the most widely applied power source in personal electronics such as digital cameras, laptops, cell phones and camcorders. Compared to other types of rechargeable batteries, Li-ion batteries have a higher voltage, lower self-discharge rate, higher energy density and no memory effect [1]. Commercial lithium ion batteries at present employ the use of graphitic carbon anode which provides a theoretical capacity of $372 \mathrm{mAh} \mathrm{g}^{-1}$ [2]. However there is a strong demand for high capacity and high energy density Li-ion batteries due to development of portable devices such as cellular phones and notebooks etc. [3]. Because of these reason, lithium metal alloys have been studied as the alternative anodes in lithium reversible batteries for many years. Among the lithium metal alloy, silicon shows a high theoretical capacity $\left(4200 \mathrm{mAh} \mathrm{g}^{-1}\right)$ of $\mathrm{Li}_{4.4} \mathrm{Si}$. But silicon anodes unavailable in practice owing to severe volume changes during lithium insertion and extraction. Many efforts have been made to solve this problem, such as incorporating inactive/active elements as cushions against volume changes [4]. Carbon has been frequently used as the active matrix because of its softness, compliance, relatively low mass, good electronic conductivity, reasonable lithium-insertion capability, and small volume expansion. Numerous methods have been employed for preparing $\mathrm{Si} / \mathrm{C}$ composite anodes, including physical mixing of silicon and graphite by ball milling, thermal vapor deposition, combined ball milling and pyrolysis, and the chemical reaction of gels [1].

In this study silicon/graphite composite anodes were produced for Li-ion batteries application by mechanical milling process. To investigate graphite effect on discharge capacity and cycling stability, different amount of graphite was used.

\section{Experimental methods}

Silicon powder $130 \mathrm{~nm}$ in size and natural graphite (average particle size $80 \mu \mathrm{m}$ ) were mechanically milled in

$80 \mathrm{ml}$ stainless bowl using $10 \mathrm{~mm}$ stainless steel balls and ball to powder weight ratio 10:1. Mechanically milling process was carried out under argon gas medium to prevent any oxidation of silicon powders by using planetary ball mill (Pulverisette 7, Fritsch).

\section{TABLE}

Weight ratio of starting materials and mechanical milling parameters.

\begin{tabular}{c|c|c}
\hline $\begin{array}{c}\text { Composition of } \\
\text { starting material [wt\%] }\end{array}$ & Samples & $\begin{array}{c}\text { Milling speed } \\
{[\mathrm{rpm}]}\end{array}$ \\
\hline 25 graphite-75 silicon & SG1 & 300 \\
50 graphite-50 silicon & SG2 & 300
\end{tabular}

Weight ratio of starting materials and mechanical milling parameters are shown in Table. Phase constituents of powders were recorded on a Rigaku Dmax 2200 X-ray diffraction (XRD) patterns technique. Surface morphologies of prepared composite electrodes were observed with JEOL 6060 LV scanning electron microscopy (SEM). For preparation of electrodes, $75 \mathrm{wt} \%$ mechanically milled active powders and 10 wt\% carbon black mixed with 15 wt\% poly(vinylidene fluorides) (PVDF) binder dissolved in $N$-methylpyrrolidinone (NMP) solution. The resulting slurry was cast on a copper foil and pasted by doctor blade coating technique and dried at $120^{\circ} \mathrm{C}$ in vacuum oven for $12 \mathrm{~h}$. Coin type CR2016 test cells were assembled in argon filled glove box, the prepared electrodes were used as a working electrode, Li foil used as counter electrode and $1 \mathrm{M} \mathrm{LiPF}_{6}$ dissolved in a mixture of ethylene carbonate (EC) and diethyl carbonate (DEC) (1:1 in volume) as the electrolyte. The working and counter electrodes were separated with polypropylene (PP) separator. Charge-discharge characteristics were obtained at $293 \mathrm{~K}$ between $80 \mathrm{mV}$ and $1.2 \mathrm{~V}$ at a constant current of $300 \mathrm{~mA} / \mathrm{g}$. 


\section{Results and discussion}

Figure 1 shows SEM micrograph of produced powders, electrodes and cross-section of electrodes surface morphology. SEM micrographs of mechanically milled powders demonstrate that silicon and graphite particles are homogeneously distributed in composite structure. Surface morphology of prepared electrodes indicates that SG2 type electrode includes more graphite powder than SG1 type electrode. Cross-sectional SEM images of electrodes represent dense electrode structure.
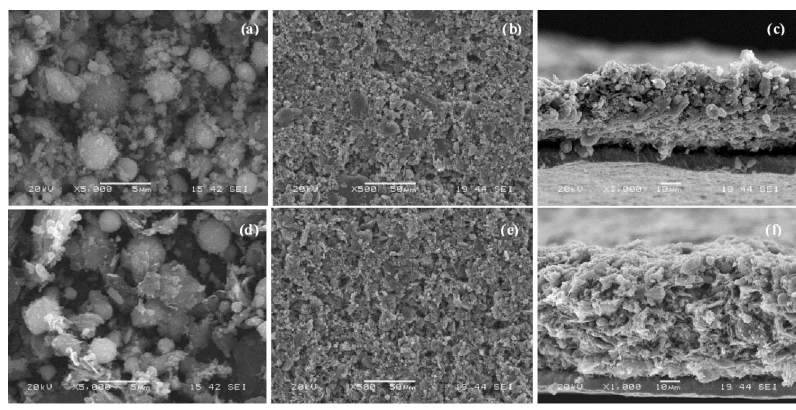

Fig. 1. SEM images of SG1 type (a) powder, (b) electrode, (c) cross-section of electrode and SG2 type (d) powder, (e) electrode, (f) cross-section of electrode.

Figure 2 shows XRD patterns of raw-silicon, SG1 type and SG2 type composite powders. When XRD patterns of graphite was investigated, graphite planes at $2 \theta$ values of approximately $26.5,45$ and $54^{\circ}$ increase with increasing amount of graphite. XRD results clearly agree with Zhang et al. [5] work.

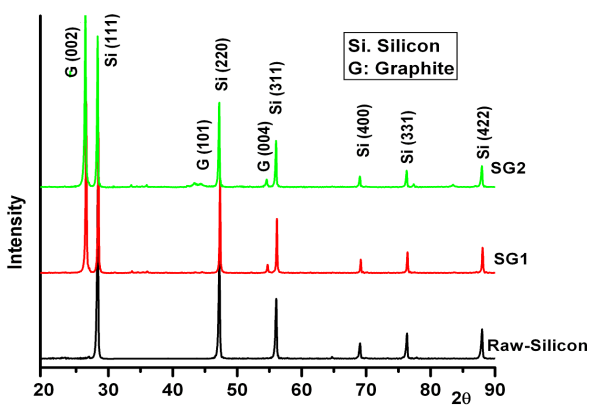

Fig. 2. XRD patterns of produced powders.

Figure 3a demonstrates cycling test curves of pure silicon anode, SG1 type composite electrode and SG2 type composite electrodes. A comparison with pure silicon in the cycling performance reveals that the SG2 type composite has a significantly enhanced cyclability as shown in Fig. 3a. According to the cycling test, best capacity retention is obtained with SG2 type composite. Therefore, charge-discharge and voltage-time profile are only investigated for SG2 type composite. The discharge capacity retention of the SG2 type composite on 50th cycle is $45 \%$ of $930 \mathrm{mAh} / \mathrm{g}$ at the first cycle. As can be seen in
Fig. $3 \mathrm{~b}$ there is a voltage plateau at about $0.1-0.2 \mathrm{~V}$ during discharge which corresponds to the alloying of Li with pure silicon. During charge process a voltage plateau is observed at about $0.4 \mathrm{~V}$ which means the dealloying of Li ions from silicon [5].

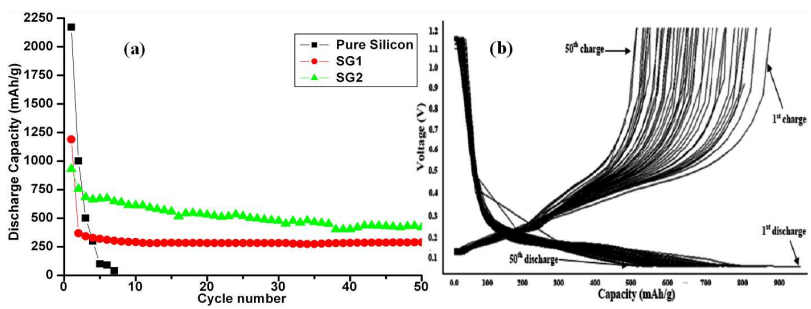

Fig. 3. (a) Cyclability and (b) voltage profile of SG2 type electrode.

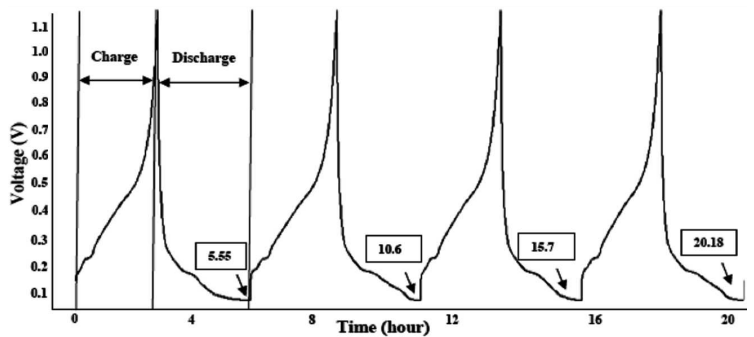

Fig. 4. Voltage-time curve of SG2 type composite electrode.

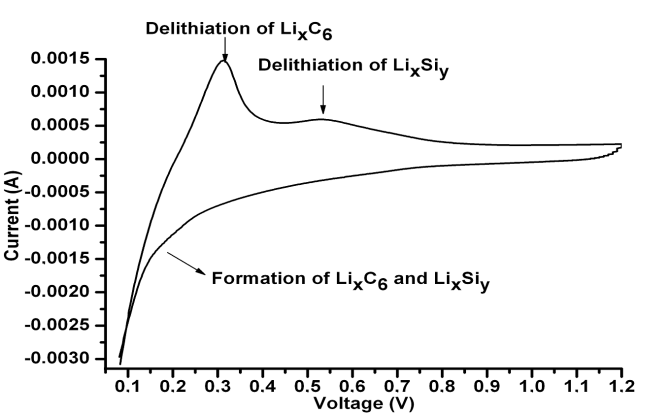

Fig. 5. Cyclic voltammetry of the first cycle SG2 type composite (scan rate $0.5 \mathrm{mV} / \mathrm{s}$ ).

Time-voltage curve of SG2 type composite up to 4 th cycle is seen in Fig. 4. Capacity retention of SG2 type composite and voltage plateaus of SG2 type composite during charge and discharge process are also seen in Fig. 4. Comparing the first cycle in the cyclic voltammetry experiment (Fig. 5), a drastic change of the currentvoltage curve and therefore electrochemical reaction of SG2 type composite with Li was clearly observed during lithium insertion and extraction. Result of cyclic voltammetry (Fig. 5) is clearly agreed with Fuchsbichler et al. [6] work. 


\section{Conclusions}

Silicon/graphite composite anode was produced by mechanical milling process. The discharge capacity retention of the SG2 type composite was found on 50th cycle is $45 \%$ of $930 \mathrm{mAh} / \mathrm{g}$ at the first cycle. SG2 type composite shows better capacity on 50th cycle than commercial graphite anode in lithium ion batteries.

\section{Acknowledgments}

This work is supported by the Scientific and Technological Research Council of Turkey (TUBITAK) under the contract number $111 \mathrm{M} 021$.

\section{References}

[1] P. Gu, R. Cai, Y. Zhou, Z. Shao, Electrochim. Acta 55, 3876 (2010).

[2] M.K. Datta, P.N. Kumta, J. Power Sources 165, 368 (2007)

[3] Q. Si, K. Hanai, T. Ichikawa, A. Hirano, N. Imanishi, Y. Takeda, O. Yamamoto, J. Power Sources 195, 1720 (2010)

[4] Q. Si, K. Hanai, N. Imanishi, M. Kubo, A. Hirano, Y. Takeda, O. Yamamoto, J. Power Sources 189, 761 (2009)

[5] Y. Zhang, X.G. Zhang, H.L. Zhang, Z.G. Zhao, F. Li, C. Liu, H.M. Cheng, Electrochim. Acta 51, 4994 (2006).

[6] B. Fuchsbichler, C. Stangl, H. Kren, F. Uhlig, S. Koller, J. Power Sources 196, 2889 (2011) 